\title{
Development and Characterization of a 400-W Slab-type Nd:YAG Gain Module
}

\author{
Yong-Ho Cha ${ }^{1 *}$, Sungman Lee ${ }^{1}$, Gwon Lim ${ }^{1}$, Sung-Hoon Baik ${ }^{1}$, Sung-Ok Kwon ${ }^{1}$, \\ Byung-Heon Cha ${ }^{1}$, Jung-Hwan $\mathrm{Lee}^{2}$, and Eung-Cheol Kang ${ }^{2}$ \\ ${ }^{1}$ Quantum Optics Division, Korea Atomic Energy Research Institute, Daejeon 305-353, Korea \\ ${ }^{2}$ Agency for Defense Development, Jochiwongil 462, Yuseong-gu, Daejeon 305-152, Korea
}

(Received December 15, 2011 : revised December 27, 2011 : accepted December 28, 2011)

\begin{abstract}
We have developed a slab-type Nd:YAG gain module based on the techniques of conduction cooling and end pumping. The Nd:YAG slab is end-capped on both ends by undoped pure YAG and is pumped through the end-caps by stacked arrays of laser diode bars. The slab's surfaces of total internal reflection are in contact on both sides with microchannel cooling blocks which are cooled by water circulation. The power oscillator based on the gain module generates more than $400 \mathrm{~W}$ at 1-kW pumping with a slope efficiency of 55\%. The small-signal gain of the gain module is 10 in a single zig-zag pass, and the amplified beam shows a near diffraction-limited beam quality.
\end{abstract}

Keywords : Nd:YAG lasers, Slab lasers

OCIS codes : (140.3530) Lasers, neodymium; (140.3280) Laser amplifiers

\section{INTRODUCTION}

High-average-power solid-state lasers with good beam quality can be used in many scientific, industrial and medical applications, and various schemes of high-power solid-state lasers have been developed. Traditionally, rod-type Nd:YAG lasers pumped by lamps or laser diodes have been studied intensively for the generation of high power lasers. Rod-type lasers, however, have not been successful in maintaining a good beam quality $\left(\mathrm{M}^{2}<5\right)$ at a high-power laser operation because of the detrimental effects of thermally induced lensing and birefringence[1-3]. The thermal lensing and birefringence are intrinsic effects which are generated when a rod-type gain material is cooled from the outer surfaces of the rod. The cooling of the rod results in a variation of the temperature in the radial direction inside the rod, and, thus, the thermal lensing effect is induced because the refractive index changes with temperature. Moreover, the stress built up in the radial and tangential direction generates the birefringence, which is not easy to compensate for. Although some schemes for the compensation of such thermal lensing and birefringence have been developed, it is still very challenging to overcome such detrimental effects at a high laser power of several hundreds of watts[4-7].

Slab-type lasers can avoid the thermal lensing and bire- fringence because of their unique heat removal scheme. The cooling of the slab's flat surfaces results in the temperature variation and the birefringence in one linear direction which is perpendicular to the cooled surfaces. Such one-dimensional birefringence yields a negligible depolarization effect for linearly polarized lasers. Moreover, the zig-zag pass by the total internal reflection (TIR) on the cooled surfaces of the slabs can minimize the optical path difference (OPD) over the laser beam profile in the zig-zag direction, which leads to significant suppression of the thermal lensing effect.

Slab-type lasers can be cooled and pumped in several ways. In early schemes, the TIR surfaces of slabs were directly water-cooled, and the pumping light was delivered through the TIR surfaces, too. The direct water-cooling and the pumping through TIR surfaces, however, led to the complicated structure of the slab-type gain module because many fittings, connections, windows and a special housing are required. To bypass the complication of the direct water cooling, a conduction-cooling scheme has been devised. The cooled surfaces of slabs are in thermal contact with cooling blocks which can be cooled simply by forced air or water circulation, and the heat generated inside the slab is transferred to the cooling blocks by conduction. Such conduction cooling schemes can have very simple and robust structure and have been successful in generating a high

\footnotetext{
*Corresponding author: yhcha@kaeri.re.kr

Color versions of one or more of the figures in this paper are available online.
} 
laser power with a good beam quality[8-10].

In this paper, we report on the development of a conductioncooled slab-type Nd:YAG gain module pumped by vertical stacks of laser diode bars (VSLD). The power oscillator built with the gain module generates more than $400 \mathrm{~W}$ of $\mathrm{cw}$ laser power with a high efficiency. The small-signal gain and the amplified beam patterns in a single zig-zag pass through the module are measured experimentally.

\section{COMPOSITION OF SLAB-TYPE GAIN MODULE}

The slab-type gain module is composed of a Nd:YAG slab, two microchannel cooling blocks, and two VSLDs, as shown in Fig. 1. The Nd:YAG slab has a size of $6 \times 2 \times$ $70 \mathrm{~mm}^{3}$ (height $\times$ thickness $\times$ length) and a composite structure of doped and undoped sections. The central 50-mm section is $0.2 \% \mathrm{Nd}$-doped and diffusion-bonded at both ends with 10-mm undoped sections. The undoped ends are angled-cut at $45^{\circ}$, and coated for anti-reflection at $1064 \mathrm{~nm}$. The TIR surfaces $\left(6 \times 70 \mathrm{~mm}^{2}\right)$ have $\sim 2$ - $\mu \mathrm{m}$-thick $\mathrm{SiO}_{2}$ coating, and the thickness of the coating is optimized for anti-reflection at $808 \mathrm{~nm}$ because the pumping laser of $808 \mathrm{~nm}$ should be injected through the TIR surfaces with minimal reflection loss. The thick $\mathrm{SiO}_{2}$ coatings also protect the TIR surfaces physically and prevent the loss of the evanescent fields of the laser beam during the reflections on TIR surfaces in contact with the metal cooling blocks.

We use two VSLDs for the pumping of the slab. The VSLD is composed of 8 laser-diode bars, and each laserdiode bar generates $65-\mathrm{W}$ laser power at $808 \mathrm{~nm}$, yielding a total laser power of $520 \mathrm{~W}$ for each VSLD. The laser beam from each laser-diode bar is collimated in both of fast and slow axis with a microlens arrays. The pumping lasers from the VSLDs are focused by cylindrical lenses in vertical and horizontal directions and injected into the slab through the TIR surfaces of the undoped ends. The focused pump beam size at the slab end is slightly smaller than the

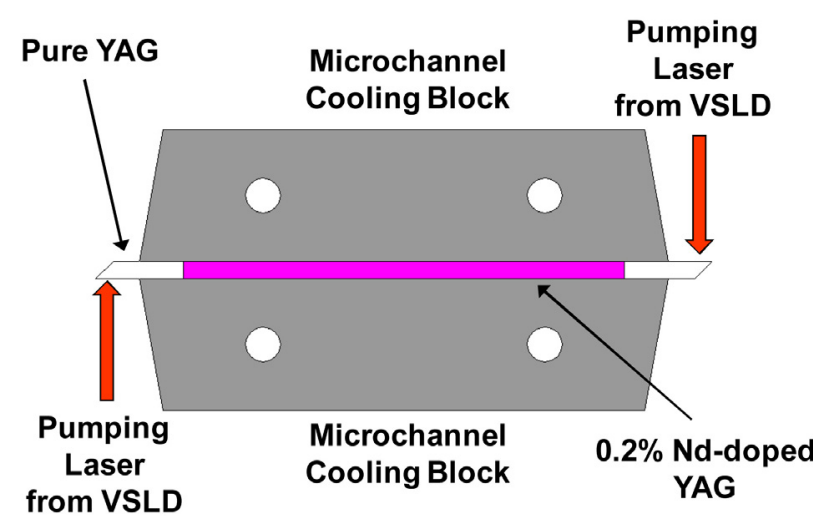

FIG. 1. Schematic of the slab-type Nd:YAG gain module with microchannel cooling blocks for conduction cooling. slab's cross section $\left(2 \times 6 \mathrm{~mm}^{2}\right)$, and, after being injected through the $45^{\circ}$-cut slab end, the pump beam is highly diverging and guided by the reflections on the slab's TIR surfaces. The pumping lasers are coupled into the central Nd-doped section of the slab as the pump beam is guided through the slab. Such end-pumping through the undoped ends confines the heat generation inside the central section which can be cooled effectively, and the thermal distortion at the ends of the Nd-doped section is reduced significantly because of the heat extraction by the undoped sections. Furthermore, the pumping profile in the slab is insensitive to the spatial distribution of diode light because of the homogenizing effect of TIR-guided propagation through the slab.

For the conduction cooling of the slabs, the TIR surfaces are in thermal contact with the microchannel cooling blocks which cover the Nd-doped sections. Indium foils are used between the slab and the cooling blocks for tight thermal contact, and the cooling blocks are cooled by water circulation. Because the cooling water is not in direct contact with the TIR surfaces, the requirement for the purity and the composition of the cooling water can be relaxed significantly compared with the direct water cooling.

\section{EXPERIMENTAL RESULTS}

To estimate the power level which can be extracted from the slab-type Nd:YAG gain module, we have built a power oscillator (PO) with the gain module, as shown in Fig. 2. The PO is composed of the gain module, two flat mirrors, and a spherical lens $(\mathrm{f}=50 \mathrm{~cm})$. One of the flat mirrors is an end-mirror with a high reflectivity at $1064 \mathrm{~nm}$, and the other flat mirror is an output coupler with a reflectivity of $30 \%$. The cavity length is $20 \mathrm{~cm}$, and the positive spherical lens is used for the stable-cavity configuration of the PO because the PO is near the boundary between stable and unstable cavities without any lens. The laser beam has a zig-zag path of 22 TIRs through the Nd:YAG slab, corresponding to the internal angle of incidence of $57.1^{\circ}$ on the TIR surfaces. In Fig. 3, the laser output power from the PO is shown with respect to the pumping power. The threshold pumping power is $200 \mathrm{~W}$, and the slope efficiency is $55 \%$, which is relatively higher compared with

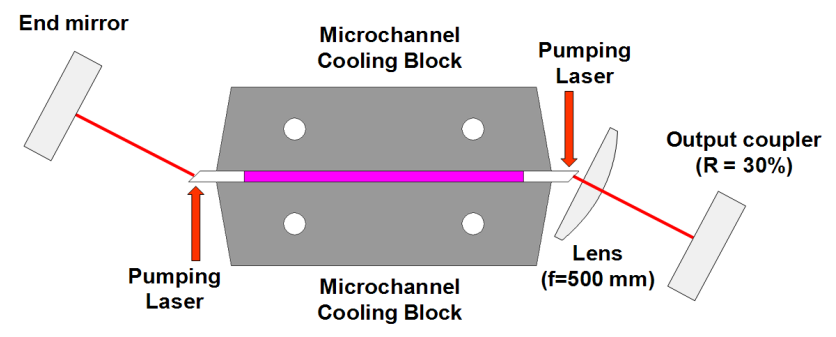

FIG. 2. Experimental setup of the power oscillator based on the slab-type Nd:YAG gain module. 


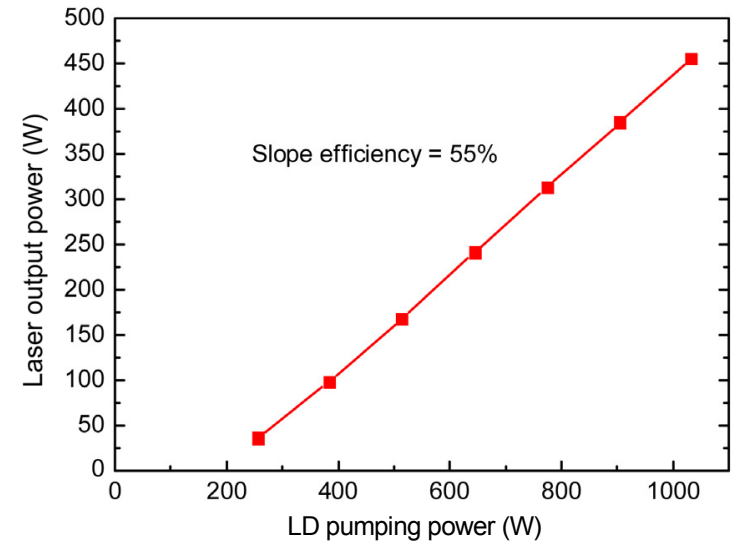

FIG. 3. Laser output power from the power oscillator based on the slab-type Nd:YAG gain module.

rod-type gain modules. Because the quantum defect and the quantum efficiency of Nd:YAG are 0.76 and $0.85[11]$, respectively, the theoretical maximal slope efficiency is about $65 \%$, assuming that the pump power is $100 \%$ absorbed in the gain medium and that there is no loss in the PO except at the output coupling. The $10 \%$ discrepancy between the theoretical and experimental results might be caused by the incomplete absorption and coupling of the pump beam. The maximal output power is limited to $450 \mathrm{~W}$ by the pumping power of $1 \mathrm{~kW}$, and the optical conversion efficiency is $45 \%$.

In many cases, gain modules are used as power amplifiers to boost up the laser power of master oscillators, and the small-signal gain is an important parameter of gain modules in the development of amplifiers. We have measured the small-signal gain of our slab-type Nd:YAG gain module by injecting a seed laser through the module. The seed laser is generated from a cw single-frequency Nd:YAG laser with a non-planar ring configuration(NPRO) coupled into a singlemode fiber. The laser output $(10 \mathrm{~mW})$ from the fiber is collimated and passed through the gain module with a zig-zag path of 22 TIRs. We measured the amplified power of the seed laser after the single zig-zag pass through the module and calculated the small-signal gain, as shown in Fig. 4. Here, the small-signal gain is defined as the ratio of the amplified power to the small input power. The smallsignal gain shows nearly exponential increase without significant gain saturation, and the small signal gain of 10 has been generated at the pumping power of $1 \mathrm{~kW}$.

For further test of the gain module as an amplifier, we set up a single-pass power amplifier with the gain module, as shown in Fig. 5. The NPRO Nd:YAG laser is preamplified to $40 \mathrm{~W}$ with a Yb-doped fiber amplifier, and the circular beam profile of the preamplified laser beam is shaped and collimated to an ellipsoidal one for the good mode match with the rectangular input aperture of the slab. Figure 6 shows that the $40-\mathrm{W}$ input laser is amplified to $190 \mathrm{~W}$ through the module at the maximal pumping power, which

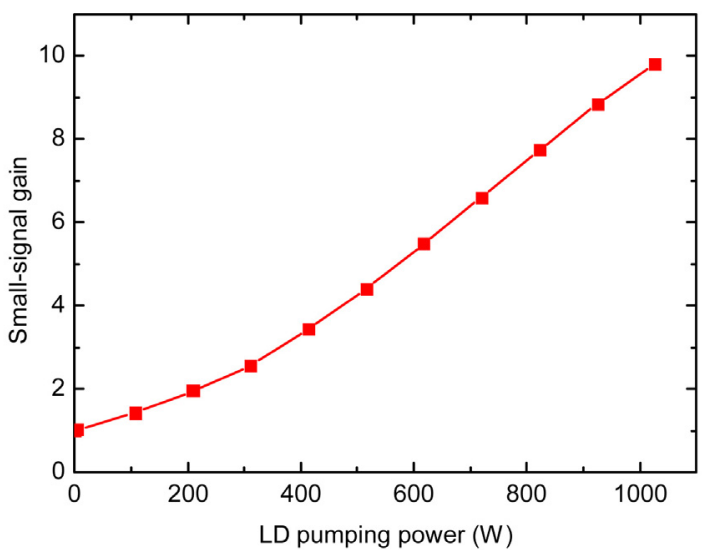

FIG. 4. Small-signal gain of the Nd:YAG gain module measured with respect to the pumping power.

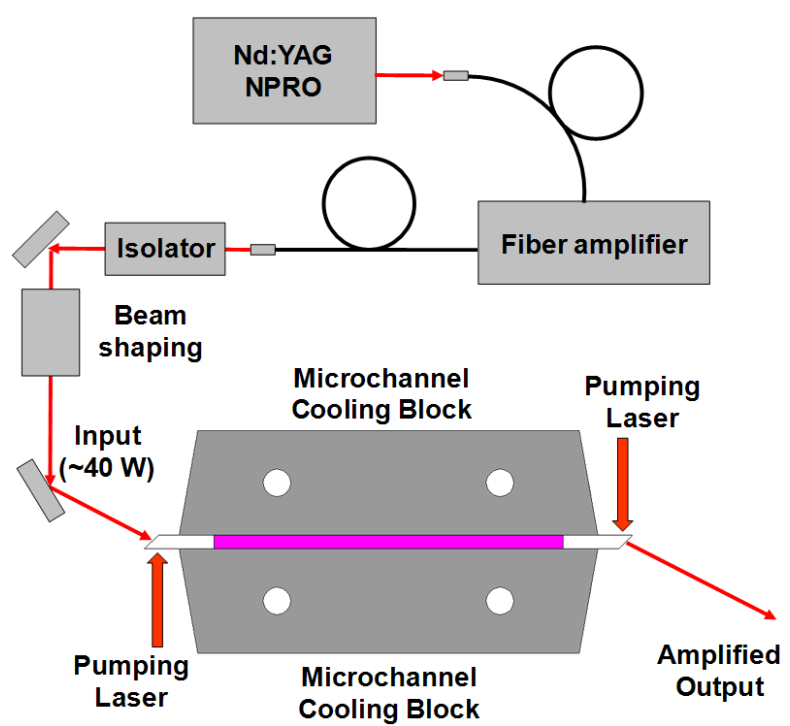

FIG. 5. Experimental setup for the single-pass power amplification with the slab-type Nd:YAG gain module.

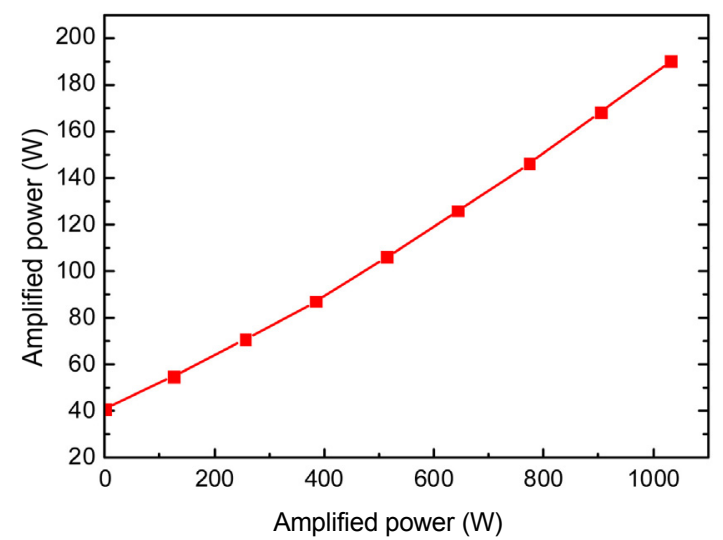

FIG. 6. Amplified output power from the Nd:YAG gain module in a single zig-zag pass configuration. The input power is $40 \mathrm{~W}$. 

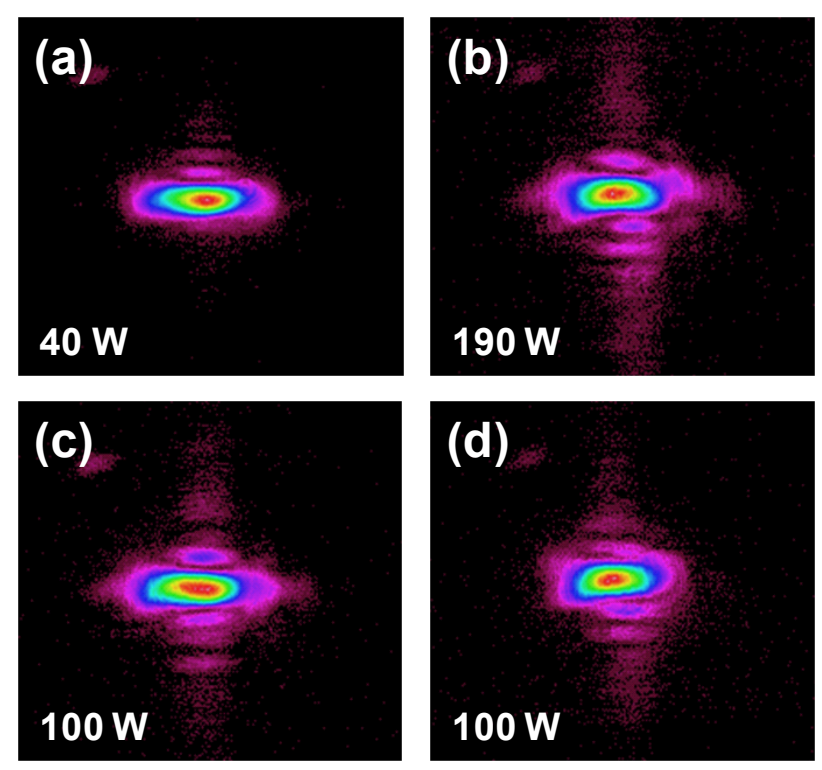

FIG. 7. Far-field beam patterns of the amplified laser beam from the Nd:YAG gain module for some pumping conditions. The amplified power is indicated in each pattern. (a) no pumping. (b) fully pumped. (c) inlet-side pumped only. (d) outlet-side pumped only.

means that $150-\mathrm{W}$ laser power is extracted in a single zig-zag pass.

Figure 7 is the far-field beam pattern of the amplified laser beam measured after the gain module. The far-field pattern shows negligible change at the highest amplified power, and, even with the asymmetric pumping of the gain module, the far-field pattern remains in a good spatial mode very close to that of the preamplified laser beam. The measured far-field beam size of the amplified laser is about 1.3 times the diffraction-limited beam size. We also measured the wanvefront of the amplified laser beam after the gain module by using a Shack-Hartmann-type wavefront sensor and found that the wavefront distortion is moderately small $(\sim 0.15$ wave RMS). This means that our gain module can be utilized effectively for the generation of high-power laser with a good beam quality. We expect that $\mathrm{M}^{2}$ of 2 or less can be achieved with a laser power of $400 \mathrm{~W}$ if we develop a hybrid stable/unstable laser cavity with our gain module[10].

\section{CONCLUSION}

In conclusion, we have developed a slab-type Nd:YAG gain module which is suitable for the generation of a highpower laser beam with a good beam quality. The Nd:YAG slab was end-pumped by VSLDs and in contact on both TIR surfaces with the microchannel cooling blocks which remove the heat of the slab by thermal conduction. From a PO built with the gain module, more than $400 \mathrm{~W}$ of laser output was generated with a slope efficiency of $55 \%$. The gain module showed a very high small-signal gain of 10 in a single zig-zag pass, and the wavefront distortion was so small that the far-field beam pattern was close to a diffraction-limited beam pattern.

\section{REFERENCES}

1. W. Koechner and D. K. Rice, "Effect of birefringence on the performance of linearly polarized YAG:Nd lasers," IEEE J. Quantum Electron. 6, 557-566 (1970).

2. W. C. Scott and M. de Wit, "Birefringence compensation and $\mathrm{TEM}_{00}$ mode enhancement in a Nd:YAG laser," Appl. Phys. Lett. 18, 3-4 (1971).

3. J. Richards, "Birefringence compensation in polarization coupled lasers," Appl. Opt. 26, 2514-2517 (1987).

4. H. J. Moon, S. M. Lee, H. S. Kim, D. K. Ko, B. H. Cha, J. H. Yi, and J. M. Lee, "Operation characteristics of diode-pumped, bifocusing compensated two-rod Nd:YAG lasers with diffusive reflectors," J. Korean Phys. Soc. 39, 881-885 (2001).

5. J. R. Park, J. Y. Lee, H. S. Kim, K. Y. Um, and H. J. Kong, "Characteristics of a birefringence compensation scheme in $\mathrm{Nd}^{3+}$ :YAG rods using a polarization rotator and imaging optics," Opt. Rev. 4, 170-175 (1997).

6. R. Bhushan, K. Tsubakimoto, H. Yoshida, H. Fujita, and M. Nakatsuka, "Simultaneous compensation of birefringence and thermal lensing effect in a high average power Nd:YAG amplifier," in Proc. CLEO/Pacific Rim 2007 (Seoul, Korea), Technical Digest WP-11, pp. 557-558.

7. Y. H. Cha, M. Y. Yang, K. H. Ko, G. Lim, J. M. Han, H. M. Park, T. S. Kim, S. Roh, and D. Y. Jeong, "270-W 15-kHz MOPA system based on side-pumped rod-type Nd:YAG gain modules," J. Opt. Soc. Korea 12, 298-392 (2008).

8. P. Zhu, D. Li, P. Hu, A. Schell, P. Shi, C. R. Haas, N. $\mathrm{Wu}$, and K. Du, "High efficiency $165 \mathrm{~W}$ near-diffractionlimited Nd:YVO4 slab oscillator pumped at $880 \mathrm{~nm}$," Opt. Lett. 33, 1930-1932 (2008).

9. A. K. Sridharan, S. Saraf, S. Sinha, and R. L. Byer, "Zigzag slabs for solid-state laser amplifiers: batch fabrication and parasitic oscillation suppression," Appl. Opt. 45, 3340-3351 (2006).

10. S. Palese, J. Harkenrider, W. Long, F. Chui, D. Hoffmaster, W. Burt, H. Injeyan, G. Conway, G. Truong, and F. Tapos, "High brightness, end-pumped, conduction cooled Nd:YAG Zig-Zag slab laser architecture," Advanced Solid State Lasers, C. Marshall, ed., Vol. 50 of OSA Trends in Optics and Photonics (Optical Society of America, USA, 2001), pp. 41-46.

11. N. P. Barnes and B. M. Walsh, "Quantum efficiency measurements of Nd:YAG, Yb:YAG, and Tm:YAG," Advanced Solid-State Lasers (Quebec, Canada, 2002), OSA TOPS vol. 68 , pp. 284-287. 